\title{
Design and analysis of ultrasonic actuator in consideration of length-reduction for a USDC (Ultrasonic/Sonic Driller/Corer)
}

\author{
Zensheu Chang, Stewart Sherrit, Mircea Badescu, Xiaoqi Bao, and Yoseph Bar-Cohen \\ Jet Propulsion Laboratory, California Institute of Technology, \\ 4800 Oak Grove Drive, Pasadena, CA 91109-8099 ${ }^{1}$
}

\begin{abstract}
Sample return and in-situ sampling and analysis is one of the major objectives of future NASA exploration missions. Existing drilling techniques are limited by the need for large axial forces, holding torques, and high power consumption. Lightweight robots and rovers have difficulties accommodating these requirements. To address these key challenges to the NASA objective of planetary in-situ rock sampling and analysis, a drilling technology called ultrasonic/sonic driller/corer (USDC) was developed. The USDC uses a novel driving mechanism, transferring ultrasonic vibration to sonic frequency impacts with the aid of a free-flying mass block (free-mass). The free mass then drives the drill bit. The actuator consists of a stack of piezoelectric disks with a horn that amplifies the induced vibration amplitudes. The standard USDC is a slender device, and some times its length is too long for specific NASA missions. It is of current interest to have novel designs that reduce the length of the device. For this purpose, two novel horn designs were examined analytically. One is the flipped horn, the other is the planar folded horn. The new designs of the horn were analyzed using finite element modeling and the results allow for the determination of the control parameters that can optimize the performance of the ultrasonic horn in terms of the tip displacement and velocity. The results of the modeling are described and discussed in this paper.
\end{abstract}

Keywords: Ultrasonic/sonic driller/corer (USDC), ultrasonic horn, planetary exploration, piezoelectric devices.

\section{INTRODUCTION}

In-situ sampling and analysis, and possibly the return of samples to earth are becoming increasingly important in NASA's space exploration missions. Existing drilling techniques are limited by the need for large axial forces and holding torques, high power consumption, large mass and inability to efficiently duty cycle. Jointly with Cybersonics, Inc., a novel USDC mechanism [Bar-Cohen et al, 2001] was developed to address these issues. The USDC mechanism is based on an ultrasonic horn actuated by a piezoelectric stack, which impacts a free-mass resonating between the horn and a drill stem, (refer to Figure 1). The ultrasonic horn is driven at its resonance frequency, ranging from $5 \mathrm{KHz}$ to 25 $\mathrm{KHz}$ depending on the specific design. The free mass bounces back and forth between the ultrasonic horn and the drill stem, converting the high frequency vibration of the horn to a hammering action with a frequency range of tens to a thousand Hz. This novel drill is capable of high-speed drilling using low axial preload and low power, and it is highly tolerant to misalignment. The USDC was demonstrated to operate from such robotic platforms as the Sojourner rover and the FIDO robotic arm and it has been shown to drill rocks as hard as granite and basalt and soft as sandstone and tuff. It has drilled 25-mm (1-inch) deep holes in granite from a 4-kg platform, 15-cm and 3-mm diameter in sandstone and $10 \mathrm{~cm}$ long, $1 \mathrm{~cm}$ diameter cores of basalt. This new USDC device is highly tolerant to changes in its operating environment, since it is driven by piezoelectric ceramics, which can be designed to operate over a wide range of temperatures including those expected on Mars as well as Venus. In Figure 2, the USDC is shown being held from its power cord while drilling a sandstone rock -- this is possible because relatively low axial preload is required.

Different applications of USDC have different requirements on the power, the dimension, the configuration of the bit, and etc. Some of NASA's missions require deep penetration into rocks, ground, or ice for sampling and analysis. Based

\footnotetext{
${ }^{1}$ Correspondence: Email zchang@ipl.nasa.gov; web: http://ndeaa.jpl.nasa.gov
} 
upon the USDC mechanism, the Ultrasonic/Sonic Gopher (USG) was developed for deep drilling [Chang et al, 2004]. Instead of using a drill bit that is long enough to reach the required depth, the USG features a coring bit with an outer diameter larger than that of the rest of the USG. So the USG can core into the target material, break the core and discard it or store it for later analysis. The drill is returned to the hole and drills, breaks and retains another core repeating the process until the required depth is reached. Some NASA's missions have a size limitation on the sampling device. It is desirable to have designs that reduce the length of the device. Quite a few novel designs of the ultrasonic horn for USDC have been proposed [Sherrit et al, 2002], aiming at reducing the length of the ultrasonic horn. In this paper, a series of analysis of these novel ultrasonic horn designs have been carried out to provide the basis for an optimal design and better understanding of these ultrasonic horns. The results will be presented and discussed in this paper.

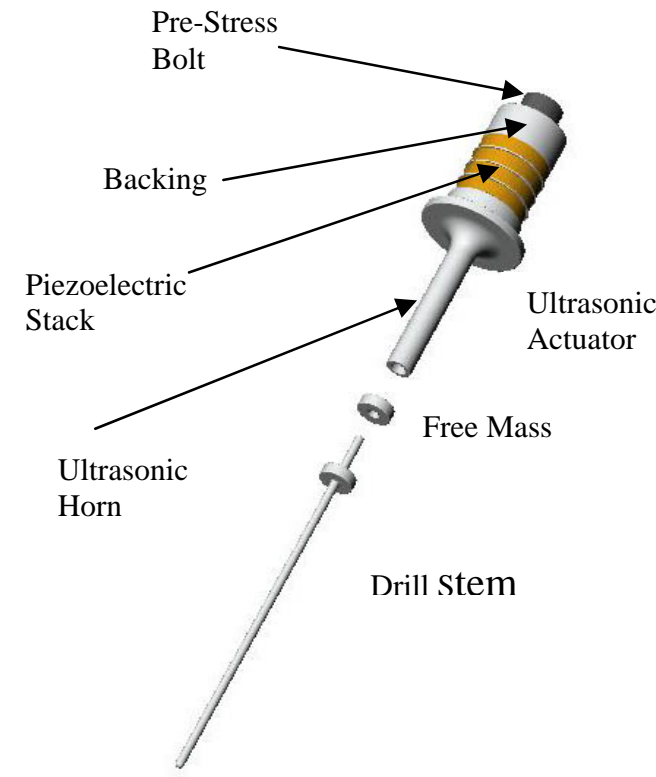

Figure 1. Exploded view of a typical USDC.

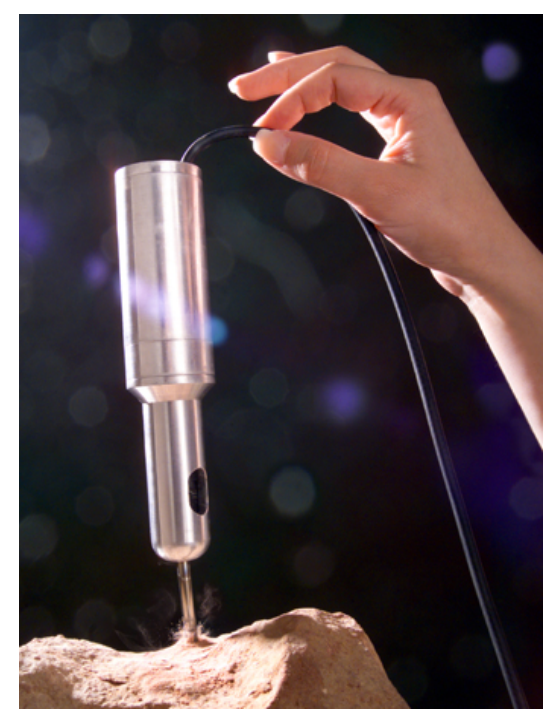

Figure 2. Ultrasonic/Sonic Driller/Corer (USDC) in action.

\section{ANALYTIC MODELING OF AN ULTRASONIC ACTUATOR}

There are three major components in a USDC [Bao et al, 2003], the ultrasonic actuator, the free mass, and the drill stem. The ultrasonic actuator is made of a stack of piezoelectric rings, an ultrasonic horn, a metal backing, and a pre-stress bolt to connect all these parts and provide pre-strain to the piezoelectric stack (Figure 1). The stack of piezoelectric material is excited at the resonance frequency of the ultrasonic actuator. Through the amplification of the ultrasonic horn, the displacement of the vibration reaches tens of microns at the tip of the horn. A free mass is contacted to the tip of the ultrasonic horn. During a drilling operation, the free mass bounces and moves back and forth between the ultrasonic horn and the drill stem. The free-mass transfers impact momentum from the ultrasonic transducer to the drill stem at a frequency ranging from tens of $\mathrm{Hz}$ to about $1000 \mathrm{~Hz}$. The shock waves caused by the impacts of the free mass upon the drill stem propagate to the bit/rock interface. The impacted brittle medium (rock, ice, etc.) is fractured when its ultimate strain is exceeded at the medium/bit interface.

The ultrasonic actuator is the most complicated component of a USDC. The design of the ultrasonic actuator determines most of the specifications of a USDC, including operating frequency, power consumption, and efficiency etc. An analytical modeling of the actuator is extremely important to determine its resonance frequency, electric impedance, electro-mechanical coupling factor, vibrating mode shape, maximum stress level, neutral plane, horn-tip displacement, etc., before eventually have it manufactured. A commercially available code ANSYS was used to perform the finite element modal and harmonic analysis. Two novel designs of ultrasonic actuator [Sherrit et al, 2002] had been 
investigated. They are the flipped horn design and the planar folded horn design [Sherrit, et al. 2001], shown in Figure 3 and 4, respectively.

Figure 3. A schematic drawing of a flipped horn.

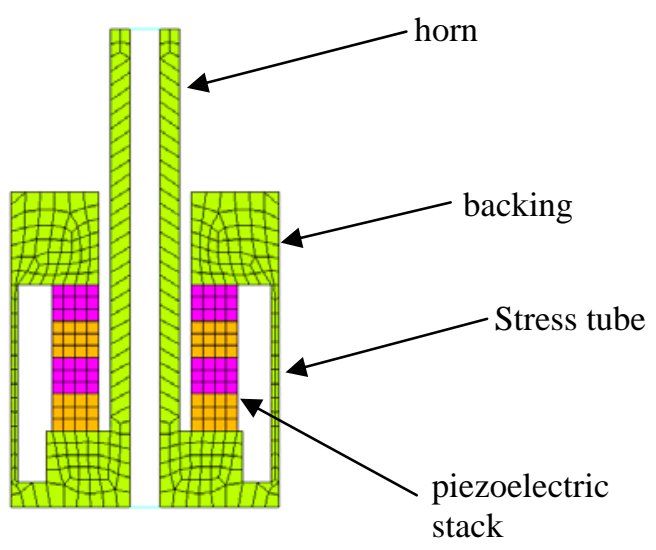

Figure 4. A 3D ANSYS model of a planar folded horn.

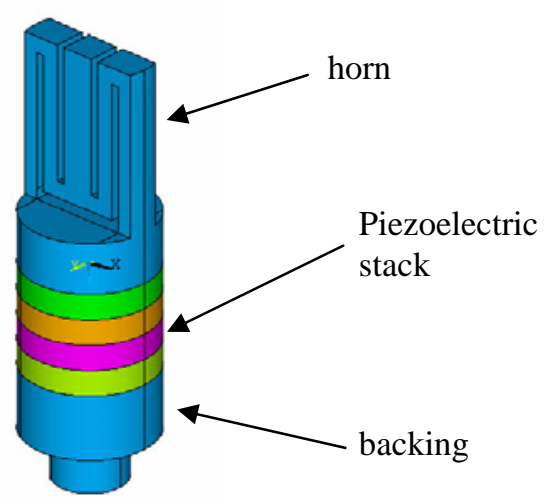

In order to study the performance of the new horn designs we first performed modal analysis on the two actuators to derive the resonance frequencies of the designed operating mode. More importantly, we need to locate the nodal point of the designed operating mode and verify if it is coincident with the designed mounting point. The nodal point is the place where the displacement remains zero while actuated. The mounting point is the place where we physically attach the ultrasonic actuator to its housing, to a drill stand, or to a robotic arm, etc. In other words, it is the interface where the actuator is connected to other structures. It is desirable to have the nodal point coincident with the mounting point. Otherwise, the whole structure will interact with the actuator and the interaction may significantly change the design resonance mode of the isolated USDC. Consequently, some energy will be lost dissipating into the whole structure and the efficiency of USDC will go down. So it is very important to match the nodal point with the mounting point.

Next, a harmonic analysis was performed by exciting the piezoelectric stack with electric field at the resonance frequency to derive the maximum horn tip displacements, the electric impedance of the actuators, and the maximum stresses in the piezoelectric stack and in the stress bolt. The horn tip displacement and the electric impedance are two of the parameters considered while selection is made between various ultrasonic horn designs. The piezoelectric material needs to be pre-strained so it doesn't break during the extension. The stress level in the piezoelectric stack is studied so we know how much pre-stress is needed while assembling the actuator. The stress bolt is usually the weakest link in the actuator assembly, and its stress level is monitored to prevent failure. 


\subsection{Modeling of the Flipped Horn}

The flipped horn is similar in shape to the standard horn, however its horn extends through the center hole of the piezoelectric stack and concentric with the stack, thereby minimizing device length without major alteration of horn design. Because the center hole of the piezoelectric stack is occupied by the horn, the stress bolt used in the standard horn is replaced by a stress tube for the flipped horn. The goal of the flipped horn design is to reduce the length of USDC by simply flipping the horn, without lowering the performance compared to its counterpart in standard horn design. It makes sense to judge the performance of a flipped horn with the standard horn as the baseline. A standard horn with a stress tube instead of stress bolt, shown in Figure 5, is modeled and analyzed. All the design parameters for the flipped and the standard horns are the same except the horn is flipped.

Figure 5. A schematic drawing of a standard horn.
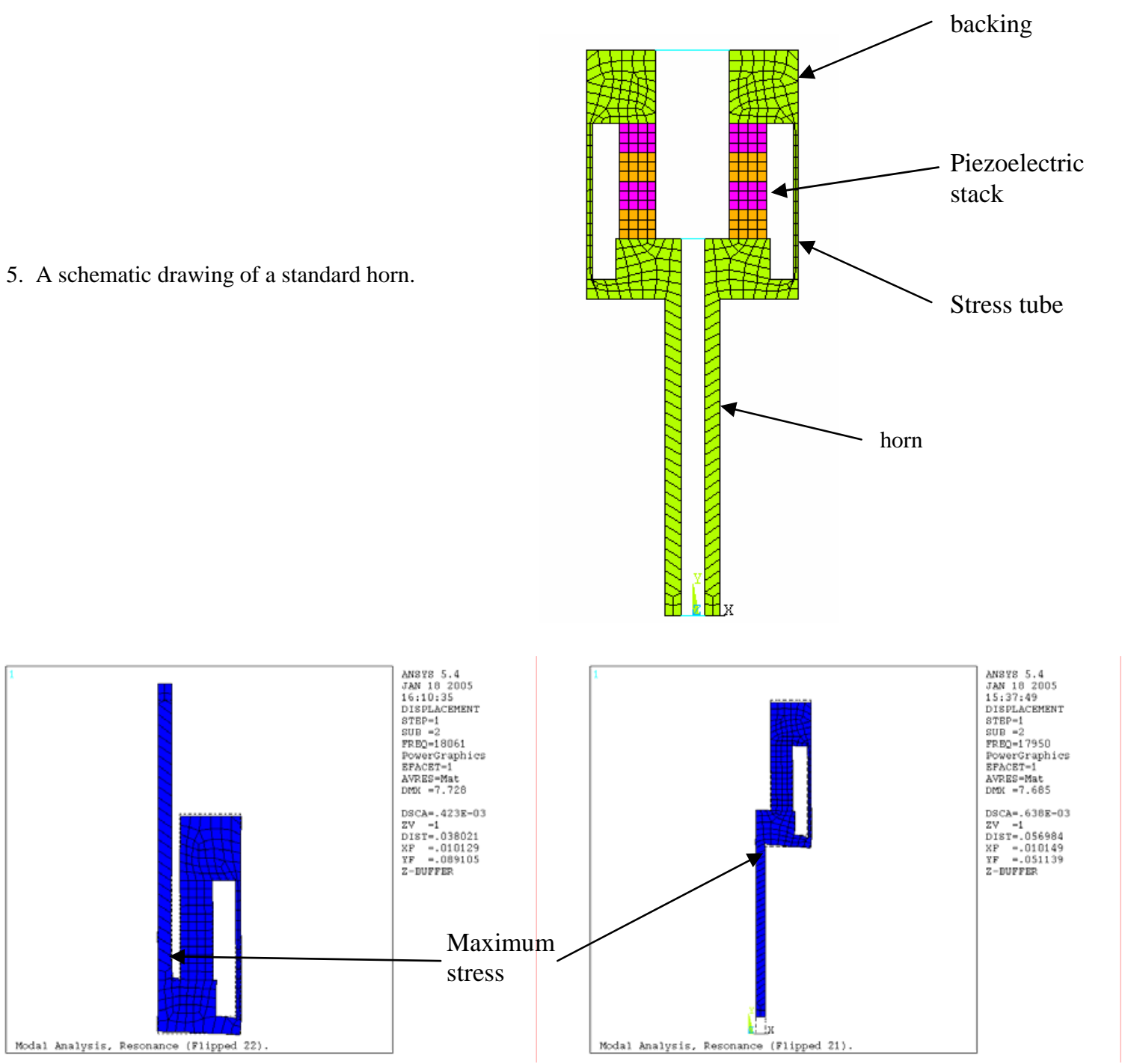

Figure 6. Result of modal analysis of the flipped horn, axissymmetric elements were used.

Figure 7. Result of modal analysis of the standard horn, axissymmetric elements were used. 
Table 1. Comparison of performance of the flipped and the standard horns.

\begin{tabular}{|c|c|c|c|}
\hline & Flipped horn & Standard horn & Difference (\%) \\
\hline Resonance frequency (Hz) & 18061 & 17950 & 0.61 \\
\hline Horn-tip disp. (m), 1 volt & $4.62 \times 10^{-7}$ & $4.63 \times 10^{-7}$ & 0.22 \\
\hline Electric current (amp), 1 volt & $1.05 \times 10^{-2}$ & $1.04 \times 10^{-2}$ & 0.95 \\
\hline Max. stress (Pa), 1 volt & $2.50 \times 10^{6}$ & $2.24 \times 10^{6}$ & 10.4 \\
\hline Horn-tip disp. (m), 1 watt & $4.51 \times 10^{-6}$ & $4.54 \times 10^{-6}$ & 0.67 \\
\hline
\end{tabular}

Modal and harmonic analysis were conducted for both the flipped and standard horns. Figures 6 and 7 show the first mode of vibration derived from modal analysis. Axis-symmetric elements were used for the analysis. It is found that the performance of the two horns is very similar. Table 1 shows the comparison of performance of the two horns. The difference in resonance frequency, horn-tip displacement and the electric current are all smaller than one percent. The maximum stress occurred on the outer surface of the horn close to the base for both cases, depicted in Figures 6 and 7 . It should be noted that the horn-tip displacement, the electric current, and the maximum stress are derived from harmonic analysis while one volt of electric field is applied to the piezoelectric stack.

\subsection{Modeling of the Planar Folded Horn}

The planar folded horn evolved from the folded horn [Sherrit et al, 2002]. Figure 8 shows a Solidworks drawing of the horn. In this design the ultrasonic horn is folded which reduce the overall length of the actuator but maintain the acoustic length. Initial experiments indicate that the tip can be adjusted by including bending displacements. By adjusting the fold thickness one can increase or decrease the bending contribution to the tip displacement. Base on this design a folded horn was fabricated. Experiments showed that the mechanical Q of the folded horn is a factor of ten below the standard horn. This has a detrimental effect on the tip displacement of the folded horn. It is also a factor of ten smaller than the standard horn. Upon close inspection it was determined that the screw threads connecting the base plate of the horn to the outer wall of the horn were the likely source of the increased dissipation of energy and reduction of resonance. The planar folded horn design provides an alternative to the folded horn. As shown in Figure 4, the planar folded horn is much easier to fabricate than the folded horn, and its base plate and folded wall are made of one piece of material which eliminates the need of the screw threads and the source of energy dissipation.
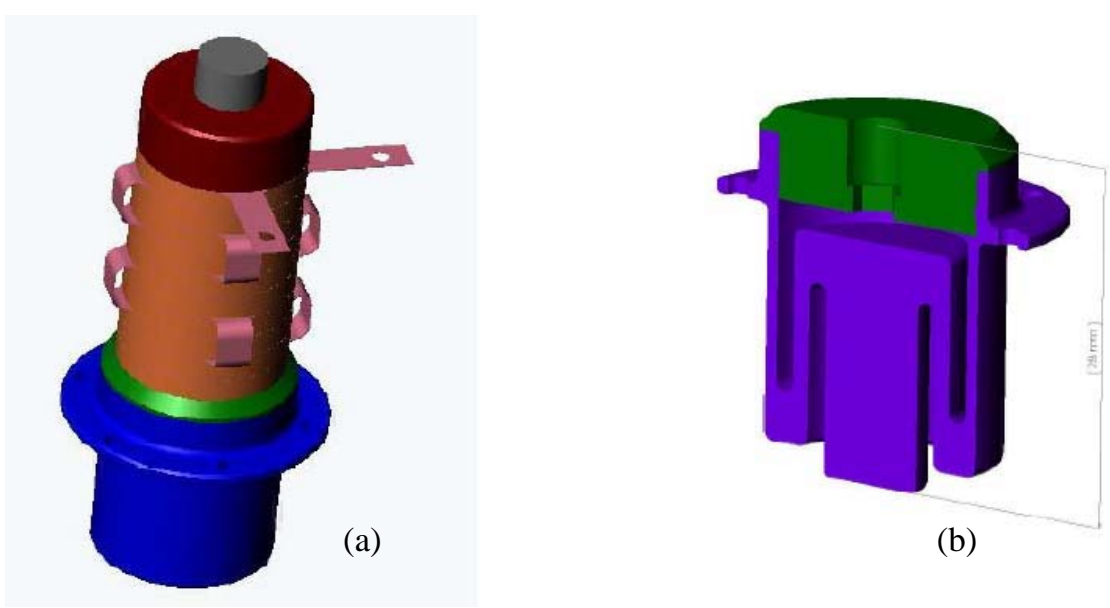

(b)

Figure 8. A Solidworks assembly drawing of the (a) ultrasonic actuator with a folded horn. (b) Cross section of the folded horn. 


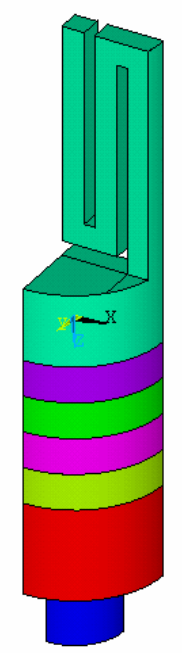

Horn length $=$
$40 \mathrm{~mm}$

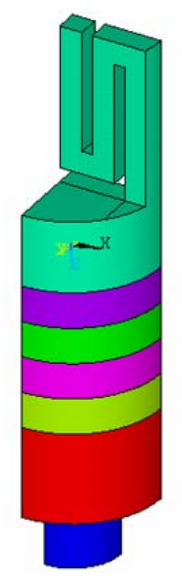

$30 \mathrm{~mm}$

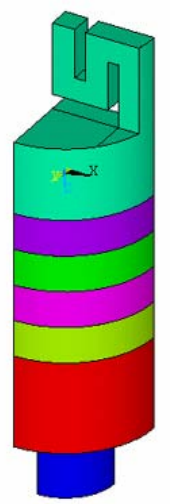

$20 \mathrm{~mm}$

Figure 9. 3-D models of ultrasonic actuators with planar folded horns of 3 different horn lengths. The actuators have 2 symmetric planes and thus only quarter of the actuators combined with symmetric boundary conditions on the two planes is needed for the models.

The planar folded horn does not have axis-symmetric feature like the folded horn design. The CPU-time economic twodimensional FEM model with axis-symmetric elements does not apply to this case, and a three-dimensional model is needed. However, it should be noted that although the planar folded horn is not axis-symmetric, it has two symmetric planes. So we only need to model a quarter of the actuator with symmetric boundary conditions applied to the two symmetric planes. Figure 9 depicts the symmetric features of the actuator and shows actuators with three different horn lengths. Since the length of the piezoelectric stack is fixed, the length of the horn becomes the dominant factor which decides the resonance frequencies of the ultrasonic actuator. To investigate the influence of the horn length on the resonance frequencies, 3 different horn lengths were used in the model. Modal analysis was performed for all the three cases and the first four vibration modes are shown in Figures 10, 11, and 12 for the horn with different lengths, respectively. Harmonic analysis was conducted for the one with horn length of $30 \mathrm{~mm}$. The results are shown in Table 2 below. For the harmonic analysis the electric field applied to the piezoelectric stack was 1 volt. The horn tip displacements were then normalized by the electric power input to derive the displacements per unit Watt.

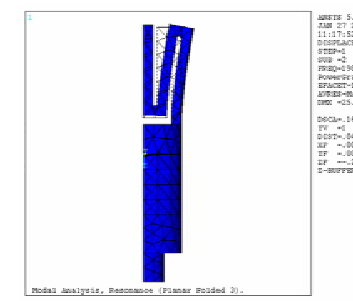

Mode 1
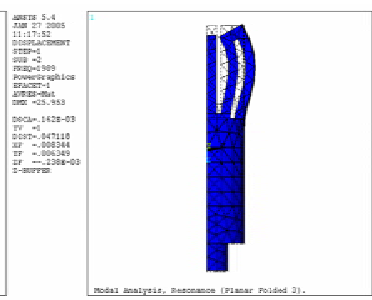

Mode 2

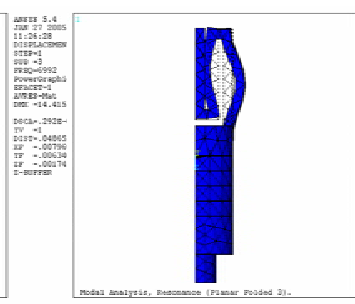

Mode 3

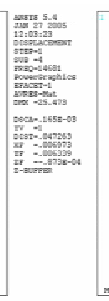

6849

14018

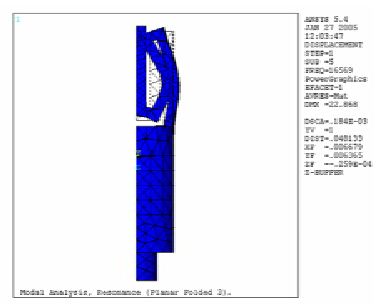

Mode 4

Resonance

Frequency (Hz)

1928

16017

Figure 10. The first 4 vibration modes derived from modal analysis of a planar folded horn with horn length of $40 \mathrm{~mm}$. 
Table 2. Results of modal and harmonic analysis for a planar folded horn with horn length of $30 \mathrm{~mm}$.

\begin{tabular}{|l|c|c|c|c|}
\hline & Mode 1 & Mode 2 & Mode 3 & Mode 4 \\
\hline Resonance Frequency (Hz) & 3693 & 11297 & 27946 & 29510 \\
\hline Tip Displacement (m), 1 Volt & $0.7029 \times 10^{-7}$ & $0.1652 \times 10^{-6}$ & $0.9226 \times 10^{-7}$ & $0.6455 \times 10^{-7}$ \\
\hline Current (Amp), 1 Volt & $0.6991 \times 10^{-4}$ & $0.5035 \times 10^{-3}$ & $0.9928 \times 10^{-2}$ & $0.1985 \times 10^{-2}$ \\
\hline Tip Displacement (m), 1 Watt & $0.8407 \times 10^{-5}$ & $0.7362 \times 10^{-5}$ & $0.9259 \times 10^{-6}$ & $0.1449 \times 10^{-5}$ \\
\hline
\end{tabular}

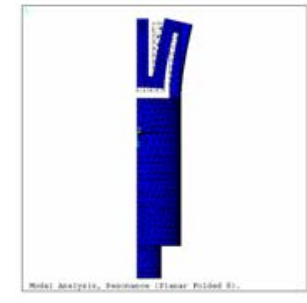

Mode 1

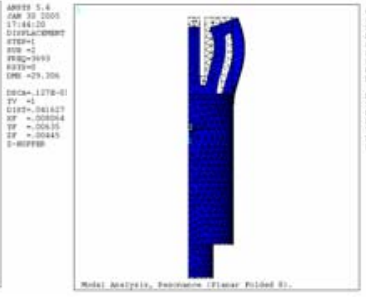

Mode 2
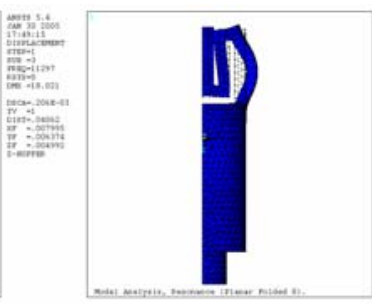

Mode 3

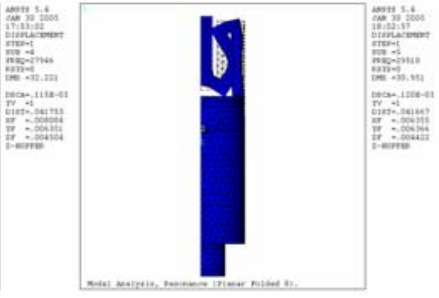

Mode 4
Resonance

Frequency $(\mathrm{Hz})$

3693

11297

27946

29510

Figure 11. The first 4 vibration modes derived from modal analysis of a planar folded horn with horn length of $30 \mathrm{~mm}$.

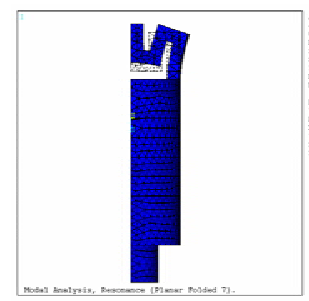

Mode 1

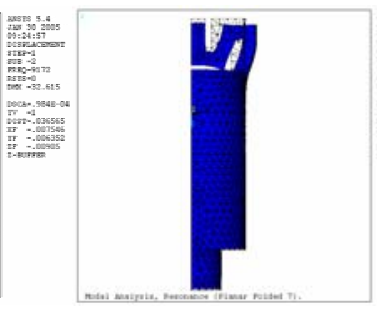

Mode 2
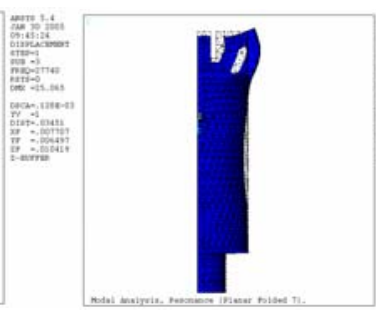

Mode 3

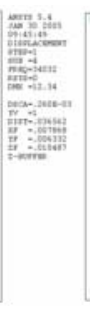

34032

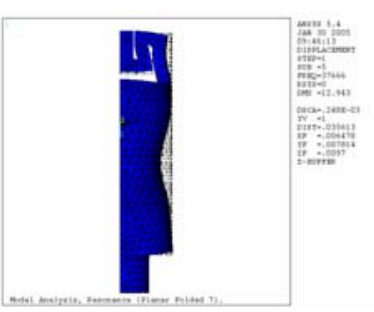

Mode 4

Resonance

Frequency $(\mathrm{Hz}) \quad 9172$

27704

37666

\section{DISCUSSION}

The analysis of the flipped horn design described in section 2.1 shows it is a promising new design which reduces the length of the ultrasonic actuator without sacrificing its performance. All the performance specifications of the flipped horn design are very similar to the standard horn design which has been proved to be working satisfactorily. The planar folded horn design has a relatively low resonance frequency for the first mode when compared to the flipped horn designs with the same piezoelectric stack, yet its horn-tip displacement is an order of magnitude higher than the flipped horn. Bending of the folded walls of the horn is the dominant factor in the resonance modes, and it seems to be the reason why the horn-tip displacement is larger. The higher modes for the planar folded horn were also investigated for possible candidate of the operating mode. However, judging by the horn-tip displacement at a unit power input, the first mode is still the best one. The ultrasonic actuators based on the two designs will be manufactured and experiments will be performed to verify the numerical results derived here. 


\section{SUMMARY}

Two-dimensional and three-dimensional Finite element analysis was conducted to study the performance of two novel horn designs, the flipped horn design and the planar folded horn design, respectively. Results show that the flipped horn design is promising based upon the facts that its performance is very similar to its counterpart in standard horn design. The planar folded horn has a relatively lower resonance frequency, yet higher horn-tip displacement compared to the flipped horn with the same piezoelectric stack. Horns based on the two designs will be fabricated and experiments will be performed to compare with the numerical results shown here.

\section{ACKNOWLEDGMENT}

Research reported in this manuscript was conducted at the Jet Propulsion Laboratory (JPL), California Institute of Technology, under a contract with National Aeronautics Space Administration (NASA).

\section{REFERENCES}

Bao X., Y. Bar-Cohen, Z. Chang, B. P. Dolgin, S. Sherrit, D. S. Pal, S. Du, and T. Peterson, "Modeling and Computer Simulation of Ultrasonic/Sonic Driller.Corer (USDC)”, IEEE Transaction on Ultrasonics, Ferroelectrics and Frequency Control (UFFC), Vol. 50, No. 9, (Sept. 2003), pp. 1147-1160.

Bar-Cohen, Y., Sherrit, S., Dolgin, B., Bao, X., Chang, Z., Krahe, R., Kroh, J., Pal, D., Du, S., and Peterson, T., "Ultrasonic/Sonic Driller/Corer(USDC) for planetary application," Proc. SPIE Smart Structure and Materials 2001, pp. 529-551, 2001.

Chang, Z., Sherrit, S., Bao, X., and Bar-Cohen, Y., "Design and Analysis of Ultrasonic Horn for USDC

(Ultrasoinc/Sonic Driller/Corer)", Proc. SPIE Smart Structure and Materials, 2004.

Sherrit, S., Askins, S.A., Gradziol M., Dolgin, B.P., Bao, X., Chang, Z., and Bar-Cohen, Y., "Novel Horn Designs for Ultrasoinc/Sonic Cleaning Welding, Soldering, Cutting and Drilling”, Proc. SPIE Smart Structure and Materials, 2002.

Sherrit S., S. A. Askins, M. Gradziel, B. P. Dolgin, Y. Bar-Cohen, X. Bao, and Z. Cheng, "Novel Ultrasonic Horns for power ultrasonics, ” NASA New Technology Report (NTR), Docket No. 30489, December 6, 2001, Patent entitled "Folded Horns for Vibration Actuators," Application \#10/612,260 Filed on July 3, 2003. 